\title{
The Pricing Dynamics of Utilities with Underdeveloped Networks
}

\author{
Omar O. Chisari, Universidad Argentina de la Empresa \\ Ioannis N. Kessides, World Bank ${ }^{1}$
}

\begin{abstract}
This paper employs an analytically tractable intertemporal framework for analyzing the dynamic pricing of a utility with an underdeveloped network (a typical case in most developing countries) facing a competitive fringe, short-run network adjustment costs, theft of service, and the threat of a retaliatory regulatory review that is increasing with the price it charges. This simple dynamic optimization model yields a number of powerful policy insights and conclusions. Under a variety of plausible assumptions (in the context of developing countries) the utility will find its long-run profits enhanced if it exercises restraint in the early stages of network development by holding price below the limit defined by the unit costs of the fringe. The utility's optimal price gradually converges toward the limit price as its network expands. Moreover, when the utility is threatened with retaliatory regulatory intervention it will generally have incentives to restrain its pricing behavior. These findings have important implications for the design of post-privatization regulatory governance in developing countries.
\end{abstract}

\section{World Bank Policy Research Working Paper 4198, April 2007}

The Policy Research Working Paper Series disseminates the findings of work in progress to encourage the exchange of ideas about development issues. An objective of the series is to get the findings out quickly, even if the presentations are less than fully polished. The papers carry the names of the authors and should be cited accordingly. The findings, interpretations, and conclusions expressed in this paper are entirely those of the authors. They do not necessarily represent the view of the World Bank, its Executive Directors, or the countries they represent. Policy Research Working Papers are available online at http://econ.worldbank.org.

\footnotetext{
${ }^{1}$ We would like to thank Daniel Benitez, Eric Groom, Atsushi Iimi, Michael Klein, Luis Serven, and Maria Vagliasindi for very helpful comments.
} 


\section{Introduction}

An important post-reform issue confronting policy makers and regulatory authorities in developing countries is how much pricing flexibility to accord to their privatized utilities to eliminate service backlogs. In many of these countries, effective demand for infrastructure services at current prices continues to substantially exceed supply, resulting in long waiting lists for service. Under these conditions, the utility's profit maximization problem is a dynamic optimization-to expand capacity at a profit-maximizing rate given short-term adjustment costs and potential diseconomies of scale to capacity expansion (marginal costs of network expansion are likely to increase as the utility reaches rural and low density areas). In formulating its various policies, including those with respect to pricing, the utility has to take into account both the current-profit and future-profit effects of those policy determinations. The two effects are generally competing with each other. For example, a price increase that is favorable to current profit will entail a sacrifice of future profits because it is likely to slow network expansion which in turn would generate future profits for the utility. Alternatively, the utility might choose to price low initially and thus sacrifice current profits for the sake of accelerating the expansion of its network and reap higher profits in the future. At the optimum, the utility's policy choice must balance the marginal increase (decrease) in current profits induced by its policy choice against the marginal decrease (increase) in future profits that the policy will entail. In the face of significant network expansion opportunities and under a variety of plausible assumptions regarding the nature of technology, costs and demand facing the infrastructure entities, dynamic optimization will lead to low pricing (relative to the short-run profit maximizing monopoly level) in the early stages of network development. Under those conditions there would be a strong rationale for light-handed regulation-i.e., for granting the infrastructure entities substantial pricing flexibility during the early stages of network development.

There is another strong rationale for light-handed regulation and for decentralizing pricing decisions to the firm level during the early post-reform years. The pursuit of pricing and other regulations to elicit optimal industrial performance in many developing countries is hindered by the lack of proper accounting systems and by the dearth of information on marginal costs, demand elasticities, and other pertinent attributes of demand and cost relationships. Under the traditional command-and-control regulatory model, any prices calculated without such information are apt to be 
inconsistent with economic efficiency and damaging to economic welfare. The information available to the firms themselves is also highly imperfect in many developing countries. Still, it seems likely that the firms will have better and more up-to-date estimates of cost and demand conditions than the regulators (Baumol and Sidak 1994). How can regulators in developing countries acquire a realistic chance of becoming effective in the face of such severe information problems? One promising policy direction would be to decentralize the decisions on pricing and other key variables to the firms that have the necessary information. The role of the regulator would be limited to imposing floors and ceilings on prices (to protect against predation and monopolistic pricing). The price-determining process then would be left to the firm, which would be free to select prices within the floor-ceiling limits. In the face of significant network expansion opportunities the firm's self-interest would motivate it to adopt prices that best serve the public interest. Such a framework could meet the dual policy objectives of giving the infrastructure entities an opportunity to obtain adequate revenue to support needed investment (an important issue in many sectors in developing countries) and protecting consumers from monopolistic pricing.

This paper presents a dynamic model of a utility in a sector with a low coverage ratio, thus facing significant network expansion opportunities but also the prospect of entry or expansion by a competitive fringe. The rate of expansion of the utility's (or equivalently the fringe's) network is assumed to vary continuously with the price set by the utility-the higher the utility's price is the more customers will join the fringe and hence the slower will be the expansion of the utility's network. Also, the utility faces the threat of retaliatory regulatory intervention that is an increasing function of the price it charges-if such regulatory intervention takes place the utility's profit is suppressed to a level that is very low relative to its short-run profit maximizing level. Moreover, a portion of the customers that leave the utility's network because of higher prices may engage in service theft-a plausible assumption in the context of many developing countries. The utility maximizes its long-run profits by balancing the impact of its pricing policy on current profits against the impact of such policy on the size of its network and the probability of regulatory intervention and hence its future profits. The utility's optimal price path depends upon its cost advantage relative to the fringe, the speed with which existing and potential customers respond to the price differential between the utility and the fringe, the discount rate, the nature of the adjustment costs that it faces with respect to network expansion, and the character of regulation. When the utility has a unit cost 
advantage over its fringe competitors, it sets low tariffs initially in order to develop its network more rapidly and thus reap larger revenues and profits in the future. As the size of the utility's network approaches its asymptotic optimum, it raises its price toward the limit defined by the unit costs of the fringe. 


\section{The Basic Model}

A common characteristic of developing countries is that a significant portion of their population, especially in periurban and rural areas, is without access to basic services. Thus, their network utilities are characterized by low coverage ratios. We consider an infrastructure sector that has not as yet achieved full coverage. A single utility firm dominates the sector serving $N$ out of $M$ potential customers. The remaining $M-N$ consumers are either being served by alternative service providers (fringe), have no access at all, or a certain portion $\rho$ of them may actually steal the service from the dominant utility through illegal connections. The services offered by the dominant utility and the fringe are assumed to be interchangeable in terms of their utility to the consumers. ${ }^{2}$

The service arrangements of the customers not connected to the utility's formal network vary by sector and country. In Yemen's electricity sector, for example, small generators supply rural towns and villages not served by the public utility. Operations range from individual households generating power for themselves and a few neighbors to units supplying up to 200 households (Ehrhardt and Burdon 1999). Electricity theft through illegal connections is a serious problem in many developing countries, especially in the inner city communities and urban areas. Electricity theft accounted for over one-quarter of total production in 1992 when privatization reforms were initiated in Argentina (Haselip 2004). In Jamaica, in excess of 30,000 illegal connections were removed each month during 2003, allowing for a monthly reduction of $4.5 \mathrm{MWh}$ of electricity production (Jamaica Public Service Company 2004). A 1989 study of the water supply in the Nigerian town of Onitsa found that water vendors delivered more water than the public water utility and that revenues for vended water exceeded revenues for water supplied by the public utility by a factor of more than 10 (Whittington et al 1991). These results indicated significant opportunities for expansion by the public utility.

\footnotetext{
${ }^{2}$ This assumption of service homogeneity is likely to be violated in many real-world circumstances. Clearly, the characteristics of the services offered by the dominant utility can differ from those offered by the fringe on several dimensions of quality. Still, in the context of many developing countries where large segments of the population remain without access to basic services, costs rather than product differentiation are likely to be the main driving force. This paper, therefore, focuses more on the nature of production technology and costs facing the fringe rather than the properties of the demand function.
} 
Let $P$ denote the average revenue generated by the utility from each customer unit and $\xi(N)$ represent the minimum unit cost of the fringe service providers. We assume that the dynamics of network expansion are characterized by the differential equation

$$
\dot{N}=\gamma[\xi(N)-P]
$$

where $\dot{N}$ is the rate of the dominant utility's customer base expansion and the coefficient $\gamma$ reflects the speed at which existing and potential customers respond to the price differential between the dominant utility and the fringe. Equation (1) implies that the rate at which customers join (or leave) the dominant utility's network varies continuously with its current price $\xi(N)$ is the limit price, i.e. that price level at which the dominant utility experiences zero network expansion. Charging less than the unit cost of the fringe $\xi(N)$ represents an investment in creating a larger network of customers which could yield dividends in terms of larger future revenues and profits. Pricing above the unit cost of the fringe, on the other hand, causes exit of customers from the utility's network and the loss of profitable network expansion opportunities. ${ }^{3}$

Let $C$ denote the utility's total cost of serving its network of $N$ customers. We allow for the possibility that a portion $\rho$ of the $M-N$ unserved customers will illegally connect to the utility's network. We further assume that

$$
C=F+c(N, \rho(M-N))
$$

where $F \geq 0$ represents the utility's fixed network costs and $c$, its variable cost function, is twice differentiable with $c_{N}>0$ and $c_{M-N} \geq 0$.

\section{Intertemporal profit maximization}

The dominant utility's long-run profit-maximization problem can be solved using the techniques of optimal control theory. The firm chooses the price $P(t)$-control variable - to maximize its profit performance functional subject to some dynamic constraints. These constraints describe the

\footnotetext{
${ }^{3}$ The dynamics of network expansion described by (1) are similar to the entry equation used by Gaskins (1971) and the vast literature that followed.
} 
evolution of the structure of the utility's network, as represented by the state variable $N(t)$,over time. Thus, the utility's long-run profit-maximization problem is given by

$$
\max _{P(t)} \Pi(0)=\int_{0}^{\infty} e^{-r t}\left[P N-C(N)-\varphi \dot{N}^{2}\right] d t
$$

subject to

$$
\begin{array}{ll}
\text { (a) } & \dot{N}=\gamma[\xi(N)-P], \\
\text { (b) } & N(0)=N_{0}, \text { given, } \\
\text { (c) } & \lim _{t \rightarrow \infty}\left[N(t) e^{-r t}\right]=0 .
\end{array}
$$

where $\Pi(0)$ is the value of the utility's profit function at time $0, r$ is the discount rate which is assumed to be constant over time, and the term $\varphi \dot{N}^{2}$ represents the adjustment costs the utility must incur in changing the size of its network (Brock and Dechert 1885; Hamermesh and Pfann 1996).

The expansion constraint in (3a) is the transition equation or equation of motion, and shows how the choice of the control variable, $P(t)$, translates into a pattern of movement for the state variable, $N(t)$. Equation (3b) simply states that the state variable $N(t)$, representing the size of the utility's network, starts at time 0 with a given value $N_{0}$.

The present value Hamiltonian of this problem is given by:

$$
H(N, P, t, \lambda)=e^{-r t} \Pi(P, N)+\lambda(t) \gamma[\xi(N)-P]
$$

where $\Pi(P, N)=P N-C(N)-\varphi \gamma^{2}[\xi(N)-P]^{2}$ and $\lambda(t)$, is the Lagrange multiplier associated with the constraint in Eq. (1). The Lagrange multiplier can be interpreted as a shadow price $-\lambda(t)$ is the imputed value to the utility of an extra customer at time $t$. The economic interpretation of the Hamiltonian in this problem is quite straightforward. The first component of the right hand side of (4) is simply the profit function at time $t$ which is dependent on the utility's pricing decision and the size of its network at that time. The second component of (4) represents the rate of change of network size which is converted to a monetary value when it is multiplied 
by the shadow price $\lambda$. At every instant in time, the utility chooses a price $P(t)$ and has a network with $N$ customers. These two variables affect the utility's objective function (profit) through two channels. First, the direct impact of $P(t)$ and $N$ on the utility's profit function is captured by the first term in (4). Second, the price $P(t)$ chosen by the utility affects the change in the size of its network in accordance to the transition equation for $\dot{N}$ in (1). The value of this change is captured by the second term in (4). Thus for a given value of the shadow price $\lambda$, the Hamiltonian captures the total impact on the utility's profit function from the choice of $P(t)$.

In terms of the present value Hamiltonian, the first-order necessary conditions for the utility's intertemporal maximization problem are given by:

$$
\begin{gathered}
H_{P}=e^{-r t}\left\{N+2 \varphi \gamma^{2}[\xi(N)-P]\right\}-\lambda \gamma=0 \\
H_{N}=e^{-r t}\left\{P-C^{\prime}(N)-2 \varphi \gamma^{2}[\xi(N)-P] \xi^{\prime}(N)\right\}+\lambda \gamma \xi^{\prime}(N)=-\dot{\lambda}
\end{gathered}
$$

These first-order conditions represent Pontryagin's Maximum Principle (Pontryagin et al 1962).

Lemma 1. If marginal network costs are increasing and $\xi^{\prime \prime}(N) \simeq 0$, then the first-order conditions (5) and (6) are sufficient for maximization iff $2 \varphi \gamma^{2}\left[C^{\prime \prime}(N)-2 \xi^{\prime}(N)\right]-1>0$.

PROOF. According to the Mangasarian Sufficiency Theorem (Mangasarian 1966), the necessary conditions of the maximum principle are also sufficient for the global maximization of the objective function $\Pi(0)$ if the Hamiltonian $H$ is concave in $(N, P)$. From (5) and (6), differentiation with respect to $N$ and $P$ yields:

$$
\begin{gathered}
H_{N N}=e^{-r t}\left\{-C^{\prime \prime}(N)-2 \varphi \gamma^{2} \xi^{\prime 2}(N)-2 \varphi \gamma^{2}[\xi(N)-P] \xi^{\prime \prime}(N)\right\}+\lambda \gamma \xi^{\prime \prime}(N) \\
H_{P P}=e^{-r t}\left(-2 \varphi \gamma^{2}\right) \\
H_{P N}=H_{N P}=e^{-r t}\left(1+2 \varphi \gamma^{2} \xi^{\prime}(N)\right) .
\end{gathered}
$$


Solving for $\lambda$ in (5) and substituting into (7) yields:

$$
H_{N N}=e^{-r t}\left[-C^{\prime \prime}(N)-2 \varphi \gamma^{2} \xi^{2}(N)+N \xi^{\prime \prime}(N)\right] \simeq e^{-r t}\left[-C^{\prime \prime}(N)-2 \varphi \gamma^{2} \xi^{\prime 2}(N)\right]
$$

If marginal network costs are increasing (i.e. $C^{\prime \prime}(N)>0$ ), then $H_{N N}<$ 0 . Also, from (8) it is clear that $H_{P P}<0$.Moreover, from (8), (9), and (10) we obtain

$$
H_{N N} H_{P P}-H_{N P}^{2}=e^{-2 r t}\left\{2 \varphi \gamma^{2}\left[C^{\prime \prime}(N)-2 \xi^{\prime}(N)\right]-1\right\} .
$$

Thus, if $2 \varphi \gamma^{2}\left[C^{\prime \prime}(N)-2 \xi^{\prime}(N)\right]-1>0$, then the Hessian of $H$ is negative definite $\left(H_{N N}<0, H_{N N} H_{P P}-H_{N P}^{2}>0\right)$, i.e. the Hamiltonian $H$ is concave in $(N, P)$. and the Mangasarian sufficiency conditions are met.

The Hamiltonian and the maximum principle that requires its maximization with respect to $P$ can obtain a more intuitively appealing economic meaning if we define the current value Hamiltonian

$$
H^{c}=\Pi(P, N)+\lambda_{c}(t) \gamma[\xi(N)-P]
$$

where $\lambda_{c}$ is the current value Lagrange multiplier. At the optimum, we obtain from the first-order conditions for maximization of $H^{c}$

$$
\frac{\partial \Pi}{\partial P}-\frac{\gamma}{r-\gamma \xi^{\prime}(N)} \frac{\partial \Pi}{\partial N}=0
$$

The first term in (13) measures the marginal increase in the current profit of the utility that is induced by an increase in its price. The second term represents the marginal decrease in future profits that such a price increase will induce via the change in the size of the utility's network-an increase in the utility's price reduces the price differential between the utility and the fringe, leading to a loss of customers to the fringe and a slower expansion in the utility's network. This effect will be larger the higher the speed $\gamma$ 
with which existing and potential customers respond to the price differential between the utility and the fringe. If the utility is myopic and discounts future profits very heavily ( $r$ is large), then it will not weigh very much in its pricing decision the impact of such a decision on the future size of its network. The utility will increase its price as long as the marginal gain in current profit made possible by such an increase is greater than the marginal decrease in future profit that its pricing decision will induce via its effect on the size of its network. The utility's optimal choice $P^{*}$ must balance these two effects.

Explicit solutions for $N, P, \lambda$ can be found by specifying $C(N)$ and $\xi(N)$. In general, it costs more to provide utility services to rural than to urban communities. The higher rural investment costs-measured as capital infrastructure costs per unit of service (e.g. kilowatt hour in the electricity sector) consumed-largely reflect the lower density of rural connections. In the electricity sector, for example, these costs depend on the community's distance from the existing medium voltage grid, and on the community's size and potential demand pattern. Investment costs per unit of demand are higher in rural areas because the bulk of demand in rural areas is for lighting during the early evening-the ratio of average demand (which determines financial and economic benefits) to peak demand (which determines investment cost) is much lower in rural systems than in urban, where there is considerable daytime electricity use. Many, if not most, developing countries are characterized by low coverage ratios and relatively underdeveloped networks with infrastructure services mainly being offered in the urban areas. In these countries, it can be reasonably assumed that the marginal costs of network expansion are increasing with the size of the network. Thus, we adopt a functional form for the dominant utility's cost function $C(N)$ that is quadratic in the size of the network $N$

$$
C(N)=F+a N+c[\rho M+(1-\rho) N]^{2}
$$

where $a>0$ and $c>0$.

We assume that the production function of the fringe exhibits increasing returns. This can be captured by positing that the minimum unit cost of the fringe service providers, $\xi(N)$, is increasing with $N$. Moreover, as the size $N$ of the formal network increases, the pool of the unserved customers $M-N$ becomes more costly to serve (assuming that the dominant utility expands service to low-cost customers first). Thus we set 


$$
\xi(N)=\xi_{0}+\xi_{1} N
$$

where $\xi_{1}>0$. We differ here from Gaskins (1971) where the fringe is assumed to have constant average and marginal cost. Where the fringe represents a small company distributing water with a truck (like in Paraguay) to periurban customers, it is reasonable to assume that because of the presence of fixed costs (e.g. the cost of capital of the truck) unit costs will increase as more customers migrate to the network of the dominant water utility-i.e. the units costs of the fringe will increase with $N$. The same would apply to a small distributed electricity company with a diesel generator offering services to customers not connected to the grid. Thus, an increase in the size of the formal network confers advantages to the dominant utility not because of the presence of positive demand externalities [e.g. Arthur (1989)] but rather because of the changes in the cost function of the fringe (which has to distribute its fixed costs over a smaller customer base).

Differentiating (5) with respect to $t$ and substituting for $\lambda$ and $\dot{\lambda}$ into (6) yields a second-order differential equation in $N$ (Euler equation)

$$
\ddot{N}-r \dot{N}-\frac{r+2 \gamma c(1-\rho)^{2}-2 \gamma \xi_{1}}{2 \varphi \gamma} N=-\gamma \frac{\xi_{0}-a-2 c \rho(1-\rho) M}{2 \varphi \gamma}
$$

whose solution is given by ${ }^{4}$

$$
N(t)=\left(N_{0}-N^{*}\right) e^{-r\left(\frac{s-1}{2}\right) t}+N^{*}
$$

where $N_{0}=N(0), \quad \zeta=\left[1+2 \frac{r+2 \gamma c(1-\rho)^{2}-2 \gamma \xi_{1}}{\varphi \gamma r^{2}}\right]^{1 / 2}$, and

$$
N^{*}=\gamma \frac{\xi_{0}-a-2 c \rho(1-\rho) M}{r+2 \gamma c(1-\rho)^{2}-2 \gamma \xi_{1}}
$$

\footnotetext{
${ }^{4}$ The general solution of (16) is given by

$N(t)=\gamma \frac{\xi_{0}-a-2 \gamma c \rho(1-\rho) M}{r+2 \gamma c(1-\rho)^{2}-2 \gamma \xi_{1}}+A e^{\omega_{1} t}+B e^{\omega_{2} t}$ where $\omega_{1}$ and $\omega_{2}$ are the roots of the quadratic equation $\omega^{2}-r \omega-\frac{r+2 \gamma c(1-\rho)^{2}-2 \gamma \xi_{1}}{2 \varphi \gamma}=0$. One of the two roots $\left(\omega_{1}\right)$ is positive, and hence it is rejected by the requirement that $N(t)$ be bounded as $t \rightarrow \infty$. The constant $B$ is determined by the initial condition $N(0)=N_{0}$ as prescribed by $(3 \mathrm{~b})$.
} 
is the steady-state size of the network. It should be noted that if the Mangasarian sufficiency conditions are met, then $\varsigma>1 .^{5}$

The optimal size of the utility's network is a function of its cost characteristics and those of the fringe, the nature of its competitive interaction with the fringe, the extent to which the utility can exclude customers for nonpayment, and the discount rate. Simple comparative static analysis indicates that the optimal size of the utility's network will be:

- bigger the larger is $\xi_{0}$, the initial unit cost (price) of the fringe;

- smaller the larger is $\alpha$, its initial marginal cost;

- bigger the more rapidly the unit costs of the fringe increase as the network absorbs more customers, i.e. the larger $\xi_{1}$ is;

- smaller the higher is $c$, the utility's marginal cost;

- smaller the higher is the utility's discount rate $r$;

- bigger the higher is the portion $\rho$ of the unserved customers who illegally connect to its network.

Equations (1) and (17) imply that the utility's optimal price path is given by

$$
P(t)=\xi_{0}+\xi_{1} N^{*}+\left(N_{0}-N^{*}\right)\left(\xi_{1}+\frac{\varsigma-1}{2}\right) e^{-r\left(\frac{\varsigma-1}{2}\right) t}
$$

with a steady-state price level

$$
P^{*}=\xi_{0}+\xi_{1} \gamma \frac{\xi_{0}-a-2 \gamma c \rho(1-\rho) M}{r+2 \gamma c(1-\rho)^{2}-2 \gamma \xi_{1}} .
$$

\footnotetext{
${ }^{5}$ According to Lemma 1, the Mangasarian sufficiency conditions are met if $2 \varphi \gamma^{2}\left[C^{\prime \prime}(N)-2 \xi^{\prime}(N)\right]-1>0 \Longrightarrow \xi_{1}<c(1-\rho)^{2}$

$-\frac{1}{4 \varphi \gamma^{2}}$ which would also imply that

$\xi_{1}<c(1-\rho)^{2}+\frac{r}{2 \gamma}$ since $\varphi, r$ and $\gamma$ are positive. This would simply imply that $r+2 \gamma c(1-\rho)^{2}-2 \gamma \xi_{1}>0$ and thus $\zeta=(1+\psi)^{1 / 2}>1$ since $\psi=2 \frac{r+2 \gamma c(1-\rho)^{2}-2 \gamma \xi_{1}}{\varphi \gamma r^{2}}>0$.
} 
The utility's terminal price is an increasing function of $\xi_{0}$ and $\xi_{1}$. Thus, it sets a higher price as the unit and marginal costs of the fringe increase.

Lemma 2. The steady state is a saddle point equilibrium.

PROOF. Differentiating (5) with respect to $t$ and substituting for $\lambda$ and $\dot{\lambda}$ into (6) lead to the differential equation in $P$

$\dot{P}=\frac{\left\{r N+2 \varphi \gamma^{2} r[\xi(N)-P]-\gamma[\xi(N)-P]-2 \varphi \gamma^{3} \xi^{\prime}(N)[\xi(N)-P]-\gamma P+\gamma C^{\prime}(N)-\gamma \xi^{\prime}(N) N\right\}}{\left(-2 \varphi \gamma^{2}\right)}$

which along with the differential equation for $N$ in (1) forms a twoequation system. To determine the properties of the steady-state equilibrium we first form the Jacobian matrix of the two-equation system and evaluate it at the steady-state point $E=\left(N^{*}, P^{*}\right)$,

$J_{E}=\left[\begin{array}{cc}\gamma \xi^{\prime}(N) & -\gamma \\ \frac{r+2 \varphi \gamma^{2} r \xi^{\prime}(N)-\gamma \xi^{\prime}(N)-2 \varphi \gamma^{3} \xi^{\prime \prime}(N)-2 \varphi \gamma^{3} \xi^{2}(N)+\gamma C^{\prime \prime}(N)-\gamma \xi^{\prime \prime}(N) N-\gamma \xi^{\prime}(N)}{\left(-2 \varphi \gamma^{2}\right)} & r-\gamma \xi^{\prime}(N)\end{array}\right]_{\left(N^{*}, P^{*}\right)}$.

The product of the two characteristic roots $\nu_{1}, \nu_{2}$ is given by

$$
\nu_{1} \nu_{2}=\left|J_{E}\right|=-\frac{r+2 \gamma c(1-\rho)^{2}-2 \gamma \xi_{1}}{2 \varphi \gamma}
$$

If the Mangasarian sufficiency conditions are met, then $r+2 \gamma c(1-\rho)^{2}-$ $2 \gamma \xi_{1}>0^{6}$ which implies that $\nu_{1} \nu_{2}<0$ and thus the steady state is locally a saddle point (Chiang 1992).

The initial size $N_{0}$ of the utility might reflect a variety of country-specific characteristics and economic policies (e.g. the country's macroeconomic condition, the socioeconomic characteristics of its population, policies related to the structure of ownership and universal access). It can also be the

\footnotetext{
${ }^{6}$ Supra note 5.
} 
unintended consequence of a host of misguided public policies (e.g. failure to prescribe cost-reflective tariffs, statutory restrictions on competition, governmental restrictions on investment) towards the sector. In many developing countries, especially during the pre-reform era, coverage ratios were very low. Large segments of the population remained with access to basic infrastructural services despite willingness to pay for such services. If the initial size $N_{0}$ of the utility's network is small, then

$$
\dot{N}(0)=-r\left(\frac{\varsigma-1}{2}\right)\left(N_{0}-N^{*}\right)>0
$$

and

$$
P_{0}=P(0)=\xi_{0}+\xi_{1} N_{0}+\left(N_{0}-N^{*}\right) \frac{\varsigma-1}{2}<\xi_{0}+\xi_{1} N_{0}
$$

Thus, the utility's initial price $P_{0}$ is lower than the limit price permitted by the initial unit cost $\xi_{0}+\xi_{1} N_{0}$ of the fringe-the utility prices initially low (below the limit price afforded by the presence of the fringe) in order to expand its network. The amount by which the utility sets its initial price below the limit price is proportional to $N_{0}-N^{*}$ (i.e. the difference between its initial and long-run optimal size) with a proportionality constant $\frac{\varsigma-1}{2}$. Given that we defined above $\zeta=\left[1+2 \frac{r+2 \gamma c(1-\rho)^{2}-2 \gamma \xi_{1}}{\varphi \gamma r^{2}}\right]^{1 / 2}$, it is easy to show that the amount by which the utility prices initially below the limit price:

- increases with $\left(\xi_{0}-a\right)$-when the dominant utility has a cost advantage over its fringe rivals, is maximizes long-run profits by setting its initial price below the limit price by an amount that is proportional to that cost advantage

- decreases with the discount rate $r$-if the utility discounts future revenues and profits very heavily then the strategy of charging less than the unit cost of the fringe in order to create a larger network of customers and reap the benefit of higher future revenues and profits is clearly less appealing; 
- decreases with $\gamma$, the speed with which existing and potential customers respond to the price differential between the dominant utility and the fringe-clearly the more responsive customers are to the price differential the smaller will be the amount by which the utility will need to underprice in order to attract a given number of customers to its network;

- decreases with $\varphi$, the rate at which the utility's adjustment costs increase with the speed with which it expands its network-the utility will have less of an incentive to price aggressively below the fringe in order to rapidly expand its network if the adjustment costs that it incurs rise rapidly with the speed of expansion;

Through simple differentiation we obtain from (19)

$$
\dot{P}(t)=-\gamma \frac{\varsigma-1}{2}\left(N_{0}-N^{*}\right)\left(\xi_{1}+\frac{\varsigma-1}{2}\right) e^{-r\left(\frac{\varsigma-1}{2}\right) t}
$$

If $N_{0}$ is small, then $\dot{P}(t)>0$, i.e. the utility follows a path of increasing tariffs.

These results could have significant implications for the design of regulatory policy in developing countries where the utility networks are underdeveloped and only a small portion of the population has access to basic infrastructural services. To the extent that the utility's intertemporal profitmaximization plan calls for a low initial price, there is no need to subject it to a detailed regulatory scrutiny, at least with respect to pricing. This is comforting because the pursuit of pricing and other regulations to elicit optimal industrial performance in many developing countries is hindered, especially during the early stages of the reform process, by the lack of proper accounting systems and by the dearth of information on marginal costs, demand elasticities, and other pertinent attributes of demand and cost relationships. Under the traditional command-and-control regulatory model, any prices calculated without such information are apt to be inconsistent with economic efficiency and be damaging to economic welfare. The information available to the firms themselves is also highly imperfect in many developing countries. Still, it seems likely that the firms will have better 
and more up-to-date estimates of cost and demand conditions than the regulators. Therefore, under those circumstances it would be appropriate to accord the utilities considerable pricing freedom to develop their networks and expand service.

\section{Pricing under endogenous regulatory threat}

One of the most urgent tasks for policy towards infrastructure industries of developing countries, especially those that have implemented privatization reforms, has been to improve the effectiveness of their regulatory frameworks. Perhaps the most serious error besetting the process has been the lack of organizational and financial independence of regulation from the politicized arms of government. Indeed, many government entities in developing countries, such as sectoral ministries, have resisted giving up their regulatory functions, especially those related to pricing. In the face of political agendas that are endemically discretionary and subject to change and reinterpretation, there can be no credible commitment to limit the discretionary powers of the regulatory apparatus. Under those institutional conditions, there exists the danger of arbitrary administrative intervention and unilateral changes in pricing policy or other regulatory directives that can substantially diminish investor value. Thus, the privatized utilities in developing countries are frequently confronted with regulatory regimes that pose significant risks of political expropriation-regulatory intervention which can substantially reduce their profits.

We analyze now the utility's pricing behavior in the face of such regulatory threat. ${ }^{7}$ Our underlying assumption is that there is a positive relationship between the price charged by the utility and the probability of retaliatory regulatory intervention. This supposition is especially relevant in the context of developing countries which have a long history of price controls, ostensibly imposed to protect the poor and to counter inflationary pressures and macroeconomic imbalances. Price levels and profits that are deemed unreasonably high by government policy makers can readily elicit retaliatory regulatory responses in the form of tighter price controls, higher profit taxes, and even threats of renationalization. In the face of endogenous regulatory threat, the utility will constrain its pricing behavior and

\footnotetext{
${ }^{7}$ The impact of regulatory threat on firm behavior has received considerable attention in the literature. These contributions include Klevorick (1973), Bawa and Sibley (1980), Logan et al (1989), Glazer and McMillan (1992), Acutt and Elliott (2001), Brunekreeft (2004), Blum et al (2006).
} 
forgo short-term profits to stave off the threatened regulation which could reduce substantially its long-term profits and market value.

Let $\Psi(t)$ the utility's assessment of the probability of regulatory intervention at time $t$, with $\Psi(0)=0$. The conditional probability density of intervention at time $t$, given its nonoccurrence prior to $t$, is $\dot{\Psi}(t) /[1-\Psi(t)]$. We assume that this conditional probability density of intervention is an increasing convex function of the utility's price $P(t)$ :

$$
\frac{\dot{\Psi}(t)}{1-\Psi(t)}=h(P(t))
$$

where $h(0)=0, h^{\prime}(P) \geq 0, h^{\prime \prime}(P) \geq 0$ (Kamien and Schwartz 1991).

The firm's objective is to maximize its expected discounted profits

$$
\max _{P(t)} \Pi(0)=\int_{0}^{\infty} e^{-r t}\{\Pi(P, N)[1-\Psi(t]+\bar{\Pi} \Psi(t)\} d t
$$

where $\Pi(P, N)=P N-C(N)-\varphi \dot{N}^{2}$

subject to

$$
\begin{array}{ll}
\text { (a) } & \dot{N}=\gamma[\xi(N)-P], \\
\text { (b) } & \dot{\Psi}(t)=[1-\Psi(t)] h(P(t)) \\
\text { (c) } & N(0)=N_{0}, \\
\text { (d) } & \lim _{t \rightarrow \infty}\left[N(t) e^{-r t}\right]=0 .
\end{array}
$$

Thus, $P$ is the control variable and $N$ and $\Psi$ are the state variables. The first component of the integrand is the utility's expected profit $\Pi$ at $t$ if there is no regulatory intervention by $t$, while the second component $\bar{\Pi}$ is the firm's reduced profit in the event it is subjected to a retaliatory regulatory scrutiny. In many developing countries, novice regulators who lack independence from sectoral ministries and the politicized arms of government, may be prone to unduly restrict the profits of privatized utilities. Under those conditions, $\bar{\Pi}$ could be substantially smaller than $\Pi$.

The current value Hamiltonian is 
$H^{c}=\Pi(P, N)[1-\Psi(t)]+\bar{\Pi} \Psi(t)+\lambda_{c}(t) \gamma[\xi(N)-P]+\mu_{c}(t) h(P(t))[1-\Psi(t)]$

where the costate variables $\lambda_{c}(t)$ and $\mu_{c}(t)$ are the current shadow prices of an extra customer and the probability of regulatory intervention. The sum of the first two terms in (29) represents the current-profit effect of the utility's pricing policy: it realizes a profit $\Pi$ if there is no regulatory intervention by $t$ (probability $1-\Psi(t))$ ) and a profit $\bar{\Pi}$ if there is regulatory intervention by $t$ (probability $\Psi(t)$ ). The third and fourth components of the Hamiltonian can be viewed as the future-profit effects of $P$ : the terms $\lambda_{c}(t) \gamma[\xi(N)-P]$ and $\mu_{c}(t) h(P(t))[1-\Psi(t)]$ represent the rate of change in the value of the utility's network and the cost of potential regulatory intervention caused by the utility's pricing decision $P$.

The maximum-principle conditions are

$$
\begin{gathered}
\frac{\partial \Pi}{\partial P}\left[1-\Psi(t]-\lambda_{c}(t) \gamma+\mu_{c}(t) h^{\prime}(P)\right)[1-\Psi(t)]=0 \\
\frac{\partial \Pi}{\partial N}[1-\Psi(t)]-\lambda_{c}(t)\left[r-\gamma \xi^{\prime}(N)\right]=-\lambda_{c}^{\prime}(t) \\
-(\Pi-\bar{\Pi})-\mu_{c}(t)[r+h(P)]=\mu_{c}^{\prime}(t) .
\end{gathered}
$$

It is easy to show that at the optimum $\frac{\lambda_{c}(t)}{1-\Psi(t)}$ and $\mu_{c}$ are constant. ${ }^{8}$ Thus, from (32) we obtain for the optimum value of $\mu_{c}$

\footnotetext{
${ }^{8}$ Differentiating (30) with respect to time yields

$\frac{\partial^{2} \Pi}{\partial P^{2}} \dot{P}[1-\Psi(t)] \quad+\quad \frac{\partial^{2} \Pi}{\partial P \partial N} \dot{N}[1-\Psi(t)] \quad-\frac{\partial \Pi}{\partial P}[1-\Psi(t)] h(P) \quad-\quad \lambda_{c}^{\prime}(t) \gamma \quad+$ $\left.\left.\mu_{c}^{\prime}(t) h^{\prime}(P)\right)[1-\Psi(t)]+\mu_{c}(t) h^{\prime \prime}(P) \dot{P}[1-\Psi(t)]-\mu_{c}(t) h(P) h^{\prime}(P)\right)[1-\Psi(t)]=0$.

In the steady state, $\dot{P}=0$ and $\dot{N}=0$. Substituting for $\lambda_{c}^{\prime}(t)$ and $\mu_{c}^{\prime}(t)$ from $(31)$ and (32) and for $\lambda_{c}(t)$ from (30) yields

$\frac{\lambda_{c}(t)}{1-\Psi(t)} \gamma^{2} \xi^{\prime}(N)=\frac{\partial \Pi}{\partial P}[r+h(P)]-\gamma \frac{\partial \Pi}{\partial N}-(\Pi-\bar{\Pi}) h^{\prime}(P)$. Thus, in the steady state $\frac{\lambda_{c}(t)}{1-\Psi(t)}$
}

is stationary. Equation (30) then implies that $\mu_{c}(t)$ is also stationary. 


$$
\mu_{c}^{*}=\frac{-(\Pi-\bar{\Pi})}{r+h(P)} .
$$

An increase in the state variable $\Psi$ raises the probability of regulatory intervention and thus reduces the optimal value at a rate proportional to the difference in profits without and with regulatory intervention.

From (30), (31), and (32) we obtain at the optimum ${ }^{9}$

$$
\frac{\partial \Pi}{\partial P}-\frac{\gamma}{r+h(P)-\gamma \xi^{\prime}(N)} \frac{\partial \Pi}{\partial N}-\frac{h^{\prime}(P)}{r+h(P)}(\Pi-\bar{\Pi})=0 .
$$

As in (13), the first term of (34) measures the marginal increase in the current profit of the utility that is induced by an increase in its price, while the second term represents the marginal decrease in future profits that such a price increase will induce via the change in the size of the utility's network. The third term measures the marginal decrease in future profits that a price increase will induce via its impact on the probability of regulatory intervention. The utility's optimal pricing policy must balance these competing effects. An increase in the utility's price increases the conditional probability of regulatory intervention at any moment which would for ever reduce the utility's profit to $\bar{\Pi}$. This effect is stronger the more sensitive is the probability of regulatory intervention to the utility's pricing behavior (i.e. the larger $h^{\prime}(P)$ is) and the more severe is the punishment imposed by regulatory intervention, i.e., the bigger is the set-back in profits $\Pi-\bar{\Pi}$. If the probability of intervention is constant (i.e. invariant with respect to the utility's behavior), then $\left.h^{\prime}(P)=0\right)$ and the third term in (34) becomes zero. Thus, the mere existence of a fixed probability of regulatory intervention does not necessarily affect the utility's behavior (Brunekreeft 2004). The constant probability of intervention simply augments $r$, the utility's discount rate. Moreover, if the utility is myopic and discounts future profits very heavily ( $r$ is large), then it will not weigh very much in its pricing decision the impact of such a decision on the probability of future regulatory intervention and hence future profits.

\footnotetext{
${ }^{9}$ In the steady state $\frac{\lambda_{c}(t)}{1-\Psi(t)}=\zeta$ or equivalently $\lambda_{c}(t)=\zeta[1-\Psi(t)]$, where $\zeta$ is a constant. This imples that $\lambda_{c}^{\prime}(t)=-\lambda_{c}(t) h(P)$. Substituting for $\lambda_{c}^{\prime}(t)$ into (31) yields $\lambda_{c}(t)=\frac{1}{r+h(P)-\gamma \xi^{\prime}(N)} \frac{\partial \Pi}{\partial N}[1-\Psi(t)]$. Finally, substituting for $\lambda_{c}(t)$ and $\mu_{c}^{*}$ into (30) yields
} $(34)$. 


\section{Regulatory threat as an expected cost}

The analysis of the utility's strategic response to regulatory threat and the calculation of its optimal price path in particular are complicated by significant nonlinearities. For the sake of analytic tractability, we choose a different way of modelling the relationship between the utility's conduct and the threat of retaliatory regulation. In the previous section we assumed that the probability of regulatory intervention is endogenously determinedit is an increasing function of the utility's price. However, the penalty suffered by the utility in the case of intervention is exogenously determinedthe utility's profits are fixed after intervention at the low level $\bar{\Pi}$. We now instead assume that the penalty of intervention is endogenously determinedit is an increasing function of the price charged by the utility and a decreasing function of the size of its network, i.e. the utility is penalized by the regulator for charging high prices and rewarded for expanding its network.

Let $\Omega(P, N)$ denote the regulatory penalty anticipated by the utility. The present value Hamiltonian in the presence of these expected regulatory penalties will be given by

$$
H(N, P, t, \lambda)=e^{-r t}\left\{P N-C(N)-\varphi \dot{N}^{2}-\Omega(P, N)\right\}+\lambda(t) \gamma[\xi(N)-P]
$$

We assume that $\Omega(P, N)$ is given by

$$
\Omega(P, N)=\lambda_{p}\left(P-P^{R}\right)+\lambda_{N}\left(N^{R}-N\right)
$$

where $\lambda_{P}>0$ and $\lambda_{N}>0$. Thus, the utility is anticipating that it will be penalized in proportion to the amount by which its price exceeds a given benchmark level $P^{R}$ and its coverage falls short a given level $N^{R}$. The benchmark levels $P^{R}$ and $N^{R}$ might be conjectural or they could be signalled by the regulator.

Let's denote with $\bar{N}^{*}$ and $\bar{P}^{*}$ the steady-state size of the utility's network and its corresponding terminal price in the presence of regulatory penalties, as contrasted with $N^{*}$ and $P^{*}$, their values when there is no regulatory intervention, given by equations (18) and (20). It is straightforward to show that $^{10}$

\footnotetext{
${ }^{10}$ We obtain the optimal path of $N$ by solving the Euler equation which is given by
} 


$$
\bar{N}^{*}=\gamma \frac{\xi_{0}-a-2 c \rho(1-\rho) M+\lambda_{P}\left(\frac{r}{\gamma}-\xi_{1}\right)+\lambda_{N}}{r+2 \gamma c(1-\rho)^{2}-2 \gamma \xi_{1}}
$$

and

$$
\bar{P}^{*}=\xi_{0}+\xi_{1} \gamma \frac{\xi_{0}-a-2 c \rho(1-\rho) M+\lambda_{P}\left(\frac{r}{\gamma}-\xi_{1}\right)+\lambda_{N}}{r+2 \gamma c(1-\rho)^{2}-2 \gamma \xi_{1}} .
$$

It is instructive to make a comparison with the case where the utility faces no regulatory penalty. Since $\frac{r}{\gamma}-\xi_{1}>0,{ }^{11}$ a comparison of equations (37) and (38) with (18) and (20) indicates that $\bar{N}^{*}>N^{*}$ and $\bar{P}^{*}>P^{*}$, i.e., the optimal size of the utility's network and its terminal price will be larger when the utility is facing regulatory penalties. We also note that $\frac{\partial \bar{N}^{*}}{\partial \lambda_{P}}=\gamma \frac{\left(\frac{r}{\gamma}-\xi_{1}\right)}{r+2 \gamma c(1-\rho)^{2}-2 \gamma \xi_{1}}>0$ and $\frac{\partial \bar{N}^{*}}{\partial \lambda_{N}}=\frac{\gamma}{r+2 \gamma c(1-\rho)^{2}-2 \gamma \xi_{1}}>0 .{ }^{12}$ Moreover, $\frac{\partial}{\partial \lambda_{P}}\left[\frac{\partial N(t)}{\partial t}\right]=r \frac{\varsigma-1}{2} \frac{\partial \bar{N}^{*}}{\partial \lambda_{P}} e^{-r\left(\frac{\varsigma-1}{2}\right) t}>0$ and $\frac{\partial}{\partial \lambda_{N}}\left[\frac{\partial N(t)}{\partial t}\right]=r \frac{\varsigma-1}{2} \frac{\partial \bar{N}^{*}}{\partial \lambda_{N}} e^{-r\left(\frac{\varsigma-1}{2}\right) t}>0$. Thus, when the utility is faced with regulatory penalties, it expands its network more rapidly. ${ }^{13}$ It is also easy to show that the utility chooses a lower initial price $\bar{P}_{0}$ than the in the case where it faces no regulatory intervention. That is, $\bar{P}_{0}=\xi_{0}+\xi_{1} N_{0}+\left(N_{0}-\bar{N}^{*}\right) \frac{\varsigma-1}{2}<P_{0}=\xi_{0}+\xi_{1} N_{0}+$ $\left(N_{0}-N^{*}\right) \frac{\varsigma-1}{2}$ because, as it was noted above, $\bar{N}^{*}>N^{*} .14$

\footnotetext{
$F_{\dot{N} \dot{N}} \ddot{N}+F_{N \dot{N}} \dot{N}+F_{t \dot{N}}-F_{N}=0 \quad$ where $F(N, \dot{N}, t)=$ $e^{-r t}\left\{\left(-\frac{1}{\gamma} \dot{N}+\xi_{0}+\xi_{1} N\right) N-F-a N-c[\rho M+(1-\rho) N]^{2}-\varphi \dot{N}^{2}-\lambda_{p}\left(P-P^{R}\right)-\lambda_{N}\left(N^{R}-N\right)\right.$. $\}$. This leads to $\ddot{N}-r \dot{N}-\frac{r+2 \gamma c(1-\rho)^{2}-2 \gamma \xi_{1}}{2 \varphi \gamma} N+\frac{\xi_{0}-a-2 c \rho(1-\rho) M+\lambda_{P}\left(\frac{r}{\gamma}-\xi_{1}\right)+\lambda_{N}}{2 \varphi}=0$ whose solution is given by $N(t)=\left(N_{0}-\bar{N}^{*}\right) e^{-r\left(\frac{\varsigma-1}{2}\right) t}+\bar{N}^{*}$ where $\bar{N}^{*}=\gamma \frac{\xi_{0}-a-2 c \rho(1-\rho) M+\lambda_{P}\left(\frac{r}{\gamma}-\xi_{1}\right)+\lambda_{N}}{r+2 \gamma c(1-\rho)^{2}-2 \gamma \xi_{1}}$ and, as before, $\zeta=\left[1+2 \frac{r+2 \gamma c(1-\rho)^{2}-2 \gamma \xi_{1}}{\varphi \gamma r^{2}}\right]^{1 / 2}$.

${ }^{11}$ This can be seen from from equation (13) where $\frac{\gamma}{r-\xi^{\prime}(N)}=\frac{1}{\frac{r}{\gamma}-\xi_{1}}$.

${ }^{12}$ This is because $r+2 \gamma c(1-\rho)^{2}-2 \gamma \xi_{1}>0$ from the Mangasarian sufficient conditions
} (supra note 4 ).

${ }^{13}$ As $\lambda_{N}$ increases, the penalty that the utility suffers for falling short the benchmark network size will increase. It is intuitively appealing then that the utility will expand its network to reduce the size of the penalty. However, we also find that the utility will expand its network when $\lambda_{P}$, the penalty that it suffers due its pricing behavior, increases. This is because the utility in maximizing its long-run profits, needs a larger network size to compensate for the loss in profit due to the pricing regulatory penalty.

${ }^{14}$ If we assume a quadratic penalty function $\Omega(P, N)=\lambda_{p}\left(P-P^{R}\right)^{2}+\lambda_{N}\left(N^{R}-\right.$ $N)^{2}$ then it is easy to show that the terminal size of the utility's network is 
The impact of the character of regulation on the utility's network expansion and price behavior should be an important consideration for policy makers in developing countries with underdeveloped networks. The imposition of a regulatory penalty has a moderating influence on the pricing behavior of a utility that pursues a long-run profit maximizing strategy by setting a low initial price in order to accelerate the development of its network and reduce the size of the regulatory penalty. Both the speed of network expansion and the utility's terminal network size will be larger when it is faced with regulatory penalties for not achieving certain network expansion benchmarks. However, while the utility's initial price is lower, its terminal price will be higher when it is faced with regulatory penalties. If we can reasonably assume that the richer customers are served first, then we can conclude that they will receive lower prices relative to the poor customers we are likely to be served after the utility expands its network. Thus, an unintended consequence of an excessively coercive regulatory policy (large $\lambda_{P}$ and $\left.\lambda_{N}\right)$ might be that it conflicts with distributional equity.

\section{The role of the discount rate}

Equations (13) and (34) illustrate the extent to which the discount rate influences the utility's choice of pricing policy which in turn has important feedback effects on the size of its network and on market structure. The basic idea of the model presented is that the utility maximizes its long-run profitability by adopting a pricing policy which at every instant balances any marginal increase on current profits against the sacrifice of future profits that such a policy might entail via its impact on the rate of the utility's network expansion and the threat of regulatory intervention. The utility's rate of time preference naturally affects the relative weights that it attaches to its current and future profits and hence the time path of prices which maximize the utility's discounted present value of profits. If the utility employs a very high discount rate it will then exercise less restraint in holding price below the fringe firm's unit cost or the short-run profit maximizing level-the value

given by $\bar{N}^{*}=\frac{\xi_{0}-a-2 c \rho(1-\rho) M+2 \lambda_{P}}{\left.\left(\frac{r}{\gamma}-\xi_{1}\right)\left(1-2 \lambda_{P} \xi_{1}\right)-P_{1}\right)\left(\frac{r}{\gamma}-\xi_{1}\right)+2 \lambda_{N} N^{R}}$. In that case then $\frac{\partial \bar{N}^{*}}{\partial \lambda_{P}}=$ $\frac{2\left(\bar{P}^{*}-P^{R}\right)\left(\frac{r}{\gamma}-\xi_{1}\right)}{\left(\frac{r}{\gamma}-\xi_{1}\right)\left(1-2 \lambda_{P} \xi_{1}\right)-\xi_{1}+2 c(1-\rho)^{2}+2 \lambda_{N}}$ and $\frac{\partial \bar{N}^{*}}{\partial \lambda_{N}}=\frac{2\left(N^{R}-\bar{N}^{*}\right)\left(\frac{r}{\gamma}-\xi_{1}\right)}{\left(\frac{r}{\gamma}-\xi_{1}\right)\left(1-2 \lambda_{P} \xi_{1}\right)-\xi_{1}+2 c(1-\rho)^{2}+2 \lambda_{N}}$ where $\bar{P}^{*}$ is again the terminal price. If the firm expects to be regulated then it is sensible to assume that $\bar{P}^{*}>P^{R}$ and $N^{R}>\bar{N}^{*}$. Since, as we noted above $\frac{r}{\gamma}-\xi_{1}>0$, then $\frac{\partial \bar{N}^{*}}{\partial \lambda_{P}}>0$ and $\frac{\partial \bar{N}^{*}}{\partial \lambda_{N}}>0$. Thus, the basic conclusions about the impact of regulatory penalty on the utility's network expansion and pricing are similar to those derived under the linear penalty function specification. 
of future profits that such a restraint will generate via its impact on the rate of the utility's network expansion decreases as the discount rate increases.

Regulation that provides a credible commitment to safeguarding the interest of both investors and customers-particularly when economic shocks create political pressure to shift the balance of power among competing interest groups-is an indispensable precondition for rational long-term planning on the part of the utilities. Unfortunately, because of their long history of arbitrary administrative intervention, governments in many developing countries lack the capacity to credibly commit against political expropriation of private value. Utilities in those countries feel especially vulnerable to unilateral changes in policy or regulatory directives that tend to diminish investor value. The fact that regulatory policy frequently lacks coherence, stability and credibility causes the utilities to employ a high discount rate in their planning. This tendency is reinforced by the uncertainty frequently underlying the macroeconomic environment in developing countries.

The initial conditions in developing countries may generate conflicting incentives on the part of their utilities with respect to pricing policy. The fact that these utilities are operating underdeveloped networks with significant expansion opportunities may provide them with powerful incentives to exercise restraint and price below the short-run profit maximizing level during the early stages of network development. The high discount rates that such utilities generally employ in the face of substantial policy uncertainty, on the other hand, will cause them to assign greater weight to current profits and consequently to set their price close to the limit defined by the fringe firms' unit costs. Therefore, if network expansion is a key goal of public policy in developing countries it is imperative that their governments undertake steps to reduce policy risk.

The rate of discount may affect the utility's price path and the extent to which it exercises pricing restraint via a different mechanism. During periods of high economic uncertainty, when the utility's discount rate is likely to increase, the regulatory agencies may be prone to intervene more aggressively in order to protect consumers or to minimize the risks that rapidly increasing utility prices may pose to a fragile economy in the midst of an economic shock. In that case, the conditional probability density of regulatory intervention might be assumed to be an increasing function of both $P$ and $r$ 


$$
\frac{\dot{\Psi}(t)}{1-\Psi(t)}=h(P, r)
$$

with $h(0,0)=0, h_{P}(P, r)>0$, and $h_{r}(P, r)>0$.

To gain some analytic tractability we assume that

$$
h(P, r)=\eta P^{\delta} r^{\varepsilon}
$$

where $\delta>0$ and $\varepsilon>0$. Equation (34) then becomes

$$
\frac{\partial \Pi_{1}}{\partial P}-\frac{\gamma}{r-\gamma \xi^{\prime}(N)} \frac{\partial \Pi_{1}}{\partial N}-\frac{\eta \delta P^{\delta-1} r^{\varepsilon}}{r+\eta P^{\delta} r^{\varepsilon}}\left(\Pi_{1}-\Pi_{2}\right)=0
$$

It is easy to show that

$$
\frac{\partial}{\partial r} \frac{\eta \delta P^{\delta-1} r^{\varepsilon}}{r+\eta P^{\delta} r^{\varepsilon}}=\frac{\eta \delta P^{\delta-1} r^{\varepsilon}(\varepsilon-1)}{\left(r+\eta P^{\delta} r^{\varepsilon}\right)^{2}} \lessgtr 0 \text { if } \varepsilon \lessgtr 1 .
$$

Thus the discount rate $r$ has two competing effects on the utility's optimal pricing policy. A higher discount rate decreases the value of future profits and thus it induces the utility to set a price that is closer to the short-run profi-maximizing level. However, a rising discount rate also increases the probability of regulatory intervention for a given choice of price. Consequently, as the discount rate increases, the utility will have to set a lower price if it is to avoid a retaliatory regulatory review. The net impact of these two competing influences depends on the magnitude of $\varepsilon$, the conditional probability density of regulatory intervention with respect to the discount rate. If the probability of regulatory intervention rises more than in proportion to the discount rate $r$ then an increase in the discount rate will cause the utility to exercise restraint with respect to pricing. This is because the increased threat of regulatory intervention associated with the higher discount rate creates an incentive for the utility to moderate its pricing, an incentive that is more powerful than the opposite one according to which a higher discount rate causes the utility to weigh its current profits more and hence to a adopt a pricing policy that maximizes short-run profits. 


\section{Concluding Comments}

We have presented in this paper an analytically tractable intertemporal framework for analyzing the dynamic pricing of a utility with an underdeveloped network (a typical case in most developing countries) facing a competitive fringe, short-run network adjustment costs, theft of service, and the threat of a regulatory review that is increasing with the price it charges. This simple dynamic optimization model yields a number of powerful policy insights and conclusions.

Under a variety of plausible assumptions (in the context of developing countries) concerning the utility's cost structure, the initial size of its network, the speed of expansion of this network in response to the price differential between the utility and the fringe, the cost structure of the competitive fringe, the costs of rapid adjustment of capacity, the percentage of customers that engage in service theft, and the probability of a retaliatory regulatory review, the utility will find its long-run profits enhanced if it exercises restraint in the early stages of network development by holding price below the limit defined by the unit costs of the fringe (or the short-run profit-maximizing level). The utility's optimal price gradually converges toward the limit price as its network expands.

These findings have important implications for the design of post privatization regulatory governance in developing countries. Many of these countries lack several institutional prerequisites for well-functioning regulatory mechanisms, including: separation of powers, especially between those of the executive and those of the judiciary; effective political and economic institutions; a well-functioning legal system; good contract law and mechanisms for resolving contract disputes; and a good supply of professional staff, expert in the relevant economic, accounting, and legal principles. In the face of scarce technical expertise, severe information problems, and lack of well-developed accounting and auditing systems, the U.S.-U.K. regulation models are likely to prove too challenging for many developing countries, especially during the early stages of the reform process. Most developing countries are ill-suited to the traditional quasi-judicial, command-andcontrol techniques of regulation, with their elaborate and complex technical and procedural requirements. More appropriate regulatory mechanisms for these countries would be simple, decentralized, flexible, and light-handed. This policy prescription for light-handed regulation during the early stages 
of the reform process is substantially reinforced by the analysis of this paper highlighting the powerful economic incentives that utilities with underdeveloped networks are likely to face during the same period to exercise restraint with respect to pricing.

The paper's intertemporal framework also highlights the critical importance of a stable, credible, and predictable policy environment for the utilities to expand their networks. If governments are able and willing to impose appropriate limits on the discretionary exercise of their regulatory powers, utilities will feel less vulnerable to unilateral changes in the policy regime that could substantially undermine their profits. Under those circumstances, utilities will tend to employ a low discount rate which effectively will cause them to set low initial prices so as to accelerate the expansion of their networks and reap higher future revenues and profits. 


\section{References}

\section{References}

[1] Acutt, M., and C. Elliott. 2001. "Threat-based Regulation and Endogenously Determined Punishments." Lancaster University Management School Working Paper 2001/007.

[2] Baumol, W., and J. Sidak. 1994. Toward Competition in Local Telephony. Cambridge, Mass.: MIT Press.

[3] Bawa, V., and D. Sibley. 1980. "Dynamic Behavior of a Firm Subject to Stochastic Regulatory Review." International Economic Review 21: 627-642.

[4] Blum, U., Growitsch, C., and N. Krap. 2006. "Network Investment and the Threat of Regulation-Preventing Monopoly Exploitation or Infrastructure Construction?" Institut für Wirtschaftsforschung Halle, Discussion Paper No. 7.

[5] Brunekreeft, G. 2004. "Regulatory Threat in Vertically Related Markets: The Case of German Electricity." European Journal of Law and Economics 17(3): 285-305.

[6] Brock, W., and W. Dechert. 1985. "Dynamic Ramsey Pricing." International Economic Review 26 (3): 569-591.

[7] Chiang, A. 1992. Dynamic Optimization. New York: McGraw-Hill, Inc.

[8] Ehrhardt, D., and R. Burdon. 1999. "Free Entry in Infrastructure." Policy Research Working Paper 2093. World Bank, Washington, D.C.

[9] Gaskins, D. 1970. "Dynamic Limit Pricing: Optimal Pricing Under Threat of Entry." Journal of Economic Theory 3: 306-322.

[10] Glazer, A., and H. McMillan. 1992. "Pricing by the Firm Under Regulatory Threat." The Quarterly Journal of Economics 107(3): 1089-1099.

[11] Hamermesh, D., and G. Pfann. 1996. "Adjustment Costs in Factor Demand." Journal of Economic Literature XXXIV: 1264-1292.

[12] Haselip, J. 2004. "The Globalization of Utilities Liberalization: Impacts Upon the Poor in Latin America." GSGR Working Paper 138/04. The University of Warwick. 
[13] Jamaica Public Service Company. 2004. What Has JPS Done to Reduce Electricity Theft? http://www.jpsco.com/site.nsf/web/electricitytheft.htm

[14] Kamien, M., and N. Schwartz. 1991. Dynamic Optimization. Amsterdam: Elsevier.

[15] Klevorick, A. 1973. "The Behavior of a Firm Subject to Stochastic Regulatory Review." Bell Journal of Economics and Management Science 4: $\quad 57-83$.

[16] Logan, J., Masson, R., and R. Reynolds. 1989. "Efficient Regulation With Little Information Reality in the Limit?" International Economic Review 30: 851-861.

[17] Mangasarian, O. 1966. "Sufficient Conditions for the Optimal Control of Nonlinear Systems." SIAM Journal on Control 4: 139-152.

[18] Pontryagin, L., Boltyanskii, V., Gamkrelidze, R., and E. Mishchenko. 1962. The Mathematical Theory of Optimal Processes. New York: Wiley.

[19] Whittington, D., Lauria, D., and X. Mu. 1991. "A Study of Water Vending and Willingness to Pay for Water in Onitsa Nigeria." World Development 19 (2): 179-198. 